\title{
Direct Adaptive Tracking Control for a Class of Pure-Feedback Stochastic Nonlinear Systems Based on Fuzzy-Approximation
}

\author{
Huanqing Wang, ${ }^{1}$ Xiaoping Liu, ${ }^{2,3}$ Qi Zhou, ${ }^{4}$ and Hamid Reza Karimi ${ }^{5}$ \\ ${ }^{1}$ School of Mathematics and Physics, Bohai University, Jinzhou, Liaoning 121000, China \\ ${ }^{2}$ Faculty of Engineering, Lakehead University, Orillia, Thunder Bay, ON, Canada P7B 5E1 \\ ${ }^{3}$ Faculty of Electronic and Information Engineering, Liaoning University of Science and Technology, Anshan, Liaoning 114051, China \\ ${ }^{4}$ College of Information Science and Technology, Bohai University, Jinzhou, Liaoning 121000, China \\ ${ }^{5}$ Department of Engineering, Faculty of Engineering and Science, University of Agder, 4898 Grimstad, Norway
}

Correspondence should be addressed to Qi Zhou; zhouqi2009@gmail.com

Received 14 November 2013; Accepted 6 January 2014; Published 13 February 2014

Academic Editor: Xiaojie Su

Copyright (c) 2014 Huanqing Wang et al. This is an open access article distributed under the Creative Commons Attribution License, which permits unrestricted use, distribution, and reproduction in any medium, provided the original work is properly cited.

The problem of fuzzy-based direct adaptive tracking control is considered for a class of pure-feedback stochastic nonlinear systems. During the controller design, fuzzy logic systems are used to approximate the packaged unknown nonlinearities, and then a novel direct adaptive controller is constructed via backstepping technique. It is shown that the proposed controller guarantees that all the signals in the closed-loop system are bounded in probability and the tracking error eventually converges to a small neighborhood around the origin in the sense of mean quartic value. The main advantages lie in that the proposed controller structure is simpler and only one adaptive parameter needs to be updated online. Simulation results are used to illustrate the effectiveness of the proposed approach.

\section{Introduction}

During the past decades, many control methods have been developed to control design of nonlinear systems, such as adaptive control [1-3], backstepping control [4], and fault tolerant control [5-9]. Particularly, backstepping-based adaptive control has been an effective tool to deal with the control problem of nonlinear strict-feedback systems without satisfying matching condition. So far, many interesting results have been obtained for deterministic nonlinear systems in $[4,10-21]$ and for stochastic cases in [22-35]. In the aforementioned papers, however, the existing results required that the nonlinear functions be in the affine forms; that is, systems are characterized by input appearing linearly in the system state equation.

Pure-feedback nonlinear systems, which have no affine appearance of the state variables that can be used as virtual control and the actual control input, stand for a more representative form than strict-feedback systems. Many practical systems are in nonaffine structure, such as biochemical process [4] and mechanical systems [36]. Therefore, the study on stability analysis and controller synthesis for pure-feedback nonlinear system is important both in theory and in practice applications [37-42]. By combining adaptive neural control and backstepping, in [37, 38], a class of pure-feedback systems was investigated, which contained only partial nonaffine functions and at least a control variable or virtual control signal existed in affine form. In [39], an "ISS-modular" approach combined with the small-gain theorem was presented for adaptive neural control of completely nonaffine pure-feedback systems. Recently, in [41], an adaptive neural tracking control scheme was presented for a class of nonaffine pure-feedback systems with multiple unknown state timevarying delays. On the other hand, it is well known that stochastic disturbance often exists in practical systems and is usually a source of instability of control systems. So, the consideration of the control design for pure-feedback nonlinear systems with stochastic disturbance is a meaningful issue and has attracted increasing attention in the control community in recent years [43-45]. In [43], the problem of adaptive fuzzy control for pure-feedback stochastic nonlinear systems has been reported. Then, Yu et al. [44] presented an adaptive 
neural controller to guarantee the four-moment boundedness for a class of uncertain nonaffine stochastic nonlinear system with time-varying delays. However, the considered systems in [43-45] are of a special kind of pure-feedback stochastic nonlinear systems in which only the last equation was viewed as nonaffine equation.

Motivated by the above observations, we will develop a novel fuzzy-based direct adaptive tracking control scheme for a class of pure-feedback stochastic nonlinear systems. The presented controller guarantees that all the signals in the closed-loop system remain bounded in probability and the tracking error converges to a small neighborhood around the origin in the sense of mean quartic value. The main contributions of this paper lie in that the structure of the proposed controller is simpler and only one adaptive parameter needs to be updated online. As a result, the computational burden is significantly alleviated and the control scheme may be more implemented in practice.

The remainder of this paper is organized as follows. The problem formulation and preliminaries are given in Section 2. An adaptive fuzzy tracking control scheme is presented in Section 3. The simulation example is given in Section 4, and it is followed by Section 5 which concludes the work.

\section{Problem Formulation and Preliminaries}

To introduce some useful conceptions and lemmas, consider the following stochastic system:

$$
d x=f(x, t) d t+h(x, t) d w, \quad \forall x \in R^{n},
$$

where $x \in R^{n}$ is state vector, $w$ is a $r$-dimensional independent standard Brownian motion defined on the complete probability space $\left(\Omega, F,\left\{F_{t}\right\}_{t \geq 0}, P\right)$ with $\Omega$ being a sample space, $F$ being a $\sigma$-field, $\left\{F_{t}\right\}_{t \geq 0}$ being a filtration, and $P$ being a probability measure, and $f: R^{n} \times R^{+} \rightarrow R^{n}, h: R^{n} \times$ $R^{+} \rightarrow R^{n \times r}$ are locally Lipschitz functions in $x$ and satisfy $f(0, t)=0, h(0, t)=0$.

Definition 1 (see [25]). For any given $V(x, t) \in C^{2,1}$, associated with the stochastic differential equation (1), define the differential operator $L$ as follows:

$$
L V=\frac{\partial V}{\partial t}+\frac{\partial V}{\partial x} f+\frac{1}{2} \operatorname{Tr}\left\{h^{T} \frac{\partial^{2} V}{\partial x^{2}} h\right\},
$$

where $\operatorname{Tr}(A)$ is the trace of $A$.

Remark 2. The term $(1 / 2) \operatorname{Tr}\left\{h^{T}\left(\partial^{2} V /\left(\partial x^{2}\right) h\right\}\right.$ is called Itô correction term, in which the second-order differential $\partial^{2} V / \partial x^{2}$ makes the controller design much more difficult than that of the deterministic system.

Definition 3 (see [46]). The trajectory $\{x(t), t \geq 0\}$ of stochastic system (1) is said to be semiglobally uniformly ultimately bounded in $p$ th moment, if, for some compact set $\Omega \in R^{n}$ and any initial state $x_{0}=x\left(t_{0}\right)$, there exist a constant $\varepsilon>0$ and a time constant $T=T\left(\varepsilon, x_{0}\right)$ such that $E\left(|x(t)|^{p}\right)<$ $\varepsilon$, for all $t>t_{0}+T$. Particularly, when $p=2$, it is usually called semiglobally uniformly ultimately bounded in mean square.
Lemma 4 (see [46]). Suppose that there exist a $C^{2,1}$ function $V(x, t): R^{n} \times R^{+} \rightarrow R^{+}$, two constants $c_{1}>0$ and $c_{2}>0$, and class $K_{\infty}$-functions $\bar{\alpha}_{1}$ and $\bar{\alpha}_{2}$ such that

$$
\begin{gathered}
\bar{\alpha}_{1}(|x|) \leq V(x, t) \leq \bar{\alpha}_{2}(|x|), \\
L V(x, t) \leq-c_{1} V(x, t)+c_{2}
\end{gathered}
$$

for all $x \in R^{n}$ and $t>t_{0}$. Then, there is a unique strong solution of system (1) for each $x_{0} \in R^{n}$ and it satisfies

$$
E[V(x, t)] \leq V\left(x_{0}\right) e^{-c_{1} t}+\frac{c_{2}}{c_{1}}, \quad \forall t>t_{0} .
$$

In this paper, we consider a class of pure-feedback stochastic nonlinear systems described by

$$
\begin{gathered}
d x_{i}=f_{i}\left(\bar{x}_{i}, x_{i+1}\right) d t+h_{i}^{T}\left(\bar{x}_{i}\right) d w, \quad 1 \leq i \leq n-1, \\
d x_{n}=f_{n}\left(\bar{x}_{n}, u\right) d t+h_{n}^{T}\left(\bar{x}_{n}\right) d w \\
y=x_{1},
\end{gathered}
$$

where $x$ and $w$ are defined in (1), $u \in R$ and $y \in R$ represent the control input and the system output, and respectively, $\bar{x}_{i}=$ $\left[x_{1}, x_{2}, \ldots, x_{i}\right]^{T} \in R^{i}, f_{i}(\cdot): R^{i+1} \rightarrow R, h_{i}(\cdot): R^{i} \rightarrow R^{r}$ $(i=1,2, \ldots, n)$ are unknown smooth nonlinear functions.

The control objective is to design a fuzzy-based adaptive tracking control law $u$ for system (5) such that the system output $y$ follows a desired reference signal $y_{d}$ in the sense of mean quartic value, and all signals in the closed-loop system remain bounded in probability. To this end, define $\bar{y}_{d}^{(i)}=$ $\left[y_{d}, y_{d}^{(1)}, \ldots, y_{d}^{(i)}\right]^{T}, i=1,2, \ldots, n$, where $y_{d}^{(i)}$ denotes the $i$ th time derivative of $y_{d}$.

For the system (5), define

$$
g_{i}\left(\bar{x}_{i}, x_{i+1}\right)=\frac{\partial f_{i}\left(\bar{x}_{i}, x_{i+1}\right)}{\partial x_{i+1}}, \quad i=1,2, \ldots, n
$$

where $x_{n+1}=u$.

Assumption 5 (see [42]). The signs of $g_{i}\left(\bar{x}_{i}, x_{i+1}\right), i=$ $1,2, \ldots, n$, are known, and there exist unknown constants $b_{m}$ and $b_{M}$ such that for $1 \leq i \leq n$,

$$
0<b_{m} \leq\left|g_{i}\left(\bar{x}_{i}, x_{i+1}\right)\right| \leq b_{M}<\infty, \quad \forall\left(\bar{x}_{i}, x_{i+1}\right) \in R^{i} \times R .
$$

Assumption 6 (see [17]). The reference signal $y_{d}(t)$ and its $n$th order derivatives are continuous and bounded.

In this note, fuzzy logic system will be used to approximate a continuous function $f(x)$ defined on some compact sets. Adopt the singleton fuzzifier, the product inference, and the center-average defuzzifier to deduce the following fuzzy rules:

$$
\begin{aligned}
& R_{i}: \text { IF } x_{1} \text { is } F_{1}^{i} \text { and } x_{2} \text { is } F_{2}^{i} \text { and } \ldots \text { and } x_{n} \text { is } F_{n}^{i} \text {. } \\
& \text { Then } y \text { is } B^{i}(i=1,2, \ldots, N),
\end{aligned}
$$


where $x=\left[x_{1}, \ldots, x_{n}\right]^{T} \in R^{n}$ and $y \in R$ are the input and output of the fuzzy system, respectively. $F_{i}^{j}$ and $B^{i}$ are fuzzy sets in $R$. Since the strategy of singleton fuzzification, centeraverage defuzzification, and product inference is used, the output of the fuzzy system can be formulated as

$$
y(x)=\frac{\sum_{j=1}^{N} \bar{W}_{j} \prod_{i=1}^{n} \mu_{F_{i}^{j}}\left(x_{i}\right)}{\sum_{j=1}^{N}\left[\prod_{i=1}^{n} \mu_{F_{i}^{j}}\left(x_{i}\right)\right]},
$$

where $\bar{W}_{j}$ is the point at which fuzzy membership function $\mu_{B^{j}}\left(\bar{W}_{j}\right)$ achieves its maximum value, which is assumed to be 1. Let

$$
s_{j}(x)=\frac{\prod_{i=1}^{n} \mu_{F_{i}^{j}}\left(x_{i}\right)}{\sum_{j=1}^{N}\left[\prod_{i=1}^{n} \mu_{F_{i}^{j}}\left(x_{i}\right)\right]},
$$

$S(x)=\left[s_{1}(x), \ldots, s_{N}(x)\right]^{T}$, and $W=\left[\bar{W}_{1}, \ldots, \bar{W}_{N}\right]^{T}$. Then, the fuzzy logic system can be rewritten as

$$
y(x)=W^{T} S(x) .
$$

If all memberships are chosen as Gaussian functions, the lemma below holds.

Lemma 7 (see [47]). Let $f(x)$ be a continuous function defined on a compact set $\Omega$. Then, for any given constant $\varepsilon>0$, there exists a fuzzy logic system (10) such that

$$
\sup _{x \in \Omega}\left|f(x)-W^{T} S(x)\right| \leq \varepsilon .
$$

The following lemma will be used in this note.

Lemma 8 (Young's inequality [23]). For $\forall(x, y) \in R^{2}$, the following inequality holds:

$$
x y \leq \frac{\varepsilon^{p}}{p}|x|^{p}+\frac{1}{q \varepsilon^{q}}|y|^{q},
$$

where $\varepsilon>0, p>1, q>1$, and $(p-1)(q-1)=1$.

\section{Adaptive Fuzzy Control Design}

In this section, a fuzzy-based adaptive tracking control scheme is proposed for the system (5). The backstepping design procedure contains $n$ steps and is developed based on the coordinate transformation $z_{i}=x_{i}-\alpha_{i-1}, i=1,2, \ldots, n$, where $\alpha_{0}=y_{d}$. The virtual control signal and the adaption law will be constructed in the following forms:

$$
\begin{gathered}
\alpha_{i}=-\frac{1}{2 a_{i}^{2}} z_{i}^{3} \widehat{\theta}, \\
\dot{\hat{\theta}}=\sum_{i=1}^{n} \frac{\sigma}{2 a_{i}^{2}} z_{i}^{6}-\gamma \widehat{\theta},
\end{gathered}
$$

where $a_{i}(i=1,2, \ldots, n), \sigma$, and $\gamma$ are positive design parameters and $Z_{1}=\left[x_{1}, y_{d}, \dot{y}_{d}\right]^{T} \in \Omega_{Z_{1}} \subset R^{3}, Z_{i}=$
$\left[\bar{x}_{i}^{T}, \widehat{\theta}, \bar{y}_{d}^{(i) T}\right]^{T} \in \Omega_{Z_{i}} \subset R^{2 i+2}(i=2, \ldots, n) . \hat{\theta}$ is the estimation of unknown constant $\theta$ which is specified as

$$
\theta=\max \left\{\frac{b_{M}^{2}}{b_{m}}\left\|W_{i}\right\|^{2} ; i=1,2, \ldots, n\right\} .
$$

Specially, $\alpha_{n}$ is the actual control input $u(t)$.

For simplicity, in the following, the time variable $t$ and the state vector $\bar{x}_{i}$ are omitted from the corresponding functions, and let $S_{i}\left(Z_{i}\right)=S_{i}$.

Step 1. Since $z_{1}=x_{1}-y_{d}$, the error dynamic satisfies

$$
d z_{1}=\left(f_{1}\left(x_{1}, x_{2}\right)-\dot{y}_{d}\right) d t+h_{1}^{T} d w .
$$

Consider a Lyapunov function candidate as

$$
V_{1}=\frac{1}{4} z_{1}^{4}+\frac{b_{m}}{2 \sigma} \widetilde{\theta}^{2}
$$

where $\widetilde{\theta}=\theta-\widehat{\theta}$ is the parameter error. By (2) and the completion of squares, one has

$$
\begin{aligned}
L V_{1} \leq & z_{1}^{3}\left(f_{1}\left(x_{1}, x_{2}\right)-\dot{y}_{d}\right)+\frac{3 z_{1}^{2}}{2} h_{1}^{T} h_{1}-\frac{b_{m}}{\sigma} \tilde{\theta} \dot{\hat{\theta}} \\
\leq & z_{1}^{3}\left(f_{1}\left(x_{1}, x_{2}\right)-\dot{y}_{d}+\frac{3}{4} l_{1}^{-2} z_{1}\left\|h_{1}\right\|^{4}\right) \\
& +\frac{3}{4} l_{1}^{2}-\frac{b_{m}}{\sigma} \tilde{\theta} \dot{\hat{\theta}},
\end{aligned}
$$

where $l_{1}$ is a constant. Define a new function

$$
w_{1}=-\dot{y}_{d}+\frac{3}{2} z_{1}+k_{1} z_{1}+\frac{3}{4} l_{1}^{-2} z_{1}\left\|h_{1}\right\|^{4},
$$

then (18) can be rewritten as

$$
\begin{aligned}
L V_{1} \leq & z_{1}^{3}\left(f_{1}\left(x_{1}, x_{2}\right)+w_{1}\right) \\
& -\left(k_{1}+\frac{3}{2}\right) z_{1}^{4}+\frac{3}{4} l_{1}^{2}-\frac{b_{m}}{\sigma} \tilde{\theta} \dot{\theta} .
\end{aligned}
$$

Based on Assumption 5 and the fact of $\partial w_{1} / \partial x_{2}=0$, one has

$$
\frac{\partial\left[f_{1}\left(x_{1}, x_{2}\right)+w_{1}\right]}{\partial x_{2}} \geq b_{m}>0 .
$$

According to Lemma 1 in [37], for each value of $x_{1}$ and $w_{1}$, there exists a smooth ideal control input $x_{2}=\bar{\alpha}_{1}\left(x_{1}, w_{1}\right)$ such that

$$
f_{1}\left(x_{1}, \bar{\alpha}_{1}\right)+w_{1}=0 .
$$

Applying mean value theorem [48], there exists $\mu_{1}\left(0<\mu_{1}<\right.$ 1) which makes

$$
f_{1}\left(x_{1}, x_{2}\right)=f_{1}\left(x_{1}, \bar{\alpha}_{1}\right)+g_{\mu_{1}}\left(x_{2}-\bar{\alpha}_{1}\right),
$$

where $g_{\mu_{1}}:=g_{1}\left(x_{1}, x_{\mu_{1}}\right)=\left.\left(\partial f_{1}\left(x_{1}, x_{2}\right) / \partial x_{2}\right)\right|_{x_{2}=x_{\mu_{1}}}, x_{\mu_{1}}=$ $\mu_{1} x_{2}+\left(1-\mu_{1}\right) \bar{\alpha}_{1}$. 
Apparently, Assumption 5 on $g_{1}\left(x_{1}, x_{2}\right)$ is still valid for $g_{\mu_{1}}$. Substituting (23) into (20) and applying the result (22) and $z_{2}=x_{2}-\alpha_{1}$ results in

$$
\begin{aligned}
L V_{1} \leq & z_{1}^{3} g_{\mu 1}\left(x_{2}-\bar{\alpha}_{1}\right)-\left(k_{1}+\frac{3}{2}\right) z_{1}^{4}+\frac{3}{4} l_{1}^{2}-\frac{b_{m}}{\sigma} \tilde{\theta} \dot{\hat{\theta}} \\
\leq & z_{1}^{3} g_{\mu_{1}} z_{2}+z_{1}^{3} g_{\mu_{1}}\left(\alpha_{1}-\bar{\alpha}_{1}\right) \\
& -\left(k_{1}+\frac{3}{2}\right) z_{1}^{4}+\frac{3}{4} l_{1}^{2}-\frac{b_{m}}{\sigma} \tilde{\theta} \dot{\hat{\theta}} .
\end{aligned}
$$

Since $\bar{\alpha}_{1}$ contains the unknown function $h_{1}, \bar{\alpha}_{1}$ cannot be directly implemented in practice. According to Lemma 7, there exists a fuzzy logic system $W_{1}^{T} S_{1}\left(Z_{1}\right)$ which can model the unknown function $\bar{\alpha}_{1}$ over a compact set $\Omega_{Z_{1}} \subset R^{3}$, such that, for any given $\varepsilon_{1}>0$,

$$
\bar{\alpha}_{1}=W_{1}^{T} S_{1}\left(Z_{1}\right)+\delta_{1}\left(Z_{1}\right), \quad\left|\delta_{1}\left(Z_{1}\right)\right| \leq \varepsilon_{1},
$$

where $\delta_{1}\left(Z_{1}\right)$ is approximation error. Then, based on Young's inequality, it follows that

$$
\begin{aligned}
-z_{1}^{3} g_{\mu_{1}} \bar{\alpha}_{1} & \leq \frac{b_{m} z_{1}^{6}}{2 a_{1}^{2}} \frac{\left\|W_{1}\right\|^{2}}{b_{m}} S_{1}^{T} S_{1}+\frac{a_{1}^{2}}{2}+\frac{3}{4} z_{1}^{4}+\frac{b_{M}^{4}}{4} \varepsilon_{1}^{4} \\
& \leq \frac{b_{m}}{2 a_{1}^{2}} z_{1}^{6} \theta+\frac{1}{2} a_{1}^{2}+\frac{3}{4} z_{1}^{4}+\frac{1}{4} b_{M}^{4} \varepsilon_{1}^{4},
\end{aligned}
$$

where the unknown constant $\theta$ is defined in (15) and the property of $S_{1}^{T} S_{1} \leq 1$ is used in (26). By choosing virtual control signal $\alpha_{1}$ in (13) with $i=1$, and using the property of $\widehat{\theta} \geq 0$ and Assumption 5, the following result holds:

$$
z_{1}^{3} g_{\mu_{1}} \alpha_{1} \leq-\frac{b_{m}}{2 a_{1}^{2}} z_{1}^{6} \widehat{\theta}
$$

Substituting (26) and (27) into (20) and using Young's inequality to the term $z_{1}^{3} g_{\mu_{1}} z_{2}$ produces

$$
L V_{1} \leq-k_{1} z_{1}^{4}+\frac{1}{4} b_{M}^{4} z_{2}^{4}+\rho_{1}+\frac{b_{m}}{\sigma} \tilde{\theta}\left(\frac{\sigma}{2 a_{1}^{2}} z_{1}^{6}-\dot{\hat{\theta}}\right)
$$

with $\rho_{1}=(1 / 2) a_{1}^{2}+(1 / 4) b_{M}^{4} \varepsilon_{1}^{4}+(3 / 4) l_{1}^{2}$.

Step 2. Based on the coordinate transformation $z_{2}=x_{2}-\alpha_{1}$ and Itô formula, one has

$$
d z_{2}=\left(f_{2}\left(\bar{x}_{2}, x_{3}\right)-\ell \alpha_{1}\right) d t+\left(h_{2}-\frac{\partial \alpha_{1}}{\partial x_{1}} h_{1}\right)^{T} d w
$$

with

$$
\ell \alpha_{1}=\frac{\partial \alpha_{1}}{\partial x_{1}} f_{1}\left(x_{1}, x_{2}\right)+\sum_{j=0}^{1} \frac{\partial \alpha_{1}}{\partial y_{d}^{(j)}} y_{d}^{(j+1)}+\frac{\partial \alpha_{1}}{\partial \widehat{\theta}} \dot{\hat{\theta}}+\frac{1}{2} \frac{\partial^{2} \alpha_{1}}{\partial x_{1}^{2}} h_{1}^{T} h_{1} .
$$

Take the following Lyapunov function:

$$
V_{2}=V_{1}+\frac{1}{4} z_{2}^{4}
$$

Then, by using a similar procedure as Step 1, it follows that

$$
\begin{aligned}
L V_{2}= & L V_{1}+z_{2}^{3}\left(f_{2}\left(\bar{x}_{2}, x_{3}\right)-\ell \alpha_{1}\right) \\
& +\frac{3}{2} z_{2}^{2}\left(h_{2}-\frac{\partial \alpha_{1}}{\partial x_{1}} h_{1}\right)^{T}\left(h_{2}-\frac{\partial \alpha_{1}}{\partial x_{1}} h_{1}\right) .
\end{aligned}
$$

It is noticed that

$$
\frac{3}{2} z_{2}^{2}\left\|h_{2}-\frac{\partial \alpha_{1}}{\partial x_{1}} h_{1}\right\|^{2} \leq \frac{3}{4 l_{2}^{2}} z_{2}^{4}\left\|h_{2}-\frac{\partial \alpha_{1}}{\partial x_{1}} h_{1}\right\|^{4}+\frac{3 l_{2}^{2}}{4},
$$

where $l_{2}$ is a constant.

Furthermore, it can be verified by substituting (28) and (33) into (32) that

$$
\begin{aligned}
L V_{2} \leq & -k_{1} z_{1}^{4}+\frac{b_{m}}{\sigma} \tilde{\theta}\left(\frac{\sigma}{2 a_{1}^{2}} z_{1}^{6}-\dot{\hat{\theta}}\right) \\
& +z_{2}^{3}\left(f_{2}\left(\bar{x}_{2}, x_{3}\right)-\ell \alpha_{1}+\frac{3 z_{2}}{4 l_{2}^{2}}\left\|h_{2}-\frac{\partial \alpha_{1}}{\partial x_{1}} h_{1}\right\|^{4}\right. \\
& \left.+\frac{1}{4} b_{M}^{4} z_{2}\right)+\rho_{1}+\frac{3}{4} l_{2}^{2} .
\end{aligned}
$$

From the definition of $\dot{\hat{\theta}}$ in (14), we have

$$
\frac{\partial \alpha_{1}}{\partial \widehat{\theta}} \dot{\hat{\theta}}=\frac{\partial \alpha_{1}}{\partial \widehat{\theta}}\left(\sum_{j=1}^{2} \frac{\sigma}{2 a_{j}^{2}} z_{j}^{6}-\gamma \widehat{\theta}\right)+\frac{\partial \alpha_{1}}{\partial \widehat{\theta}} \sum_{j=3}^{n} \frac{\sigma}{2 a_{j}^{2}} z_{j}^{6} .
$$

By combining (30), (34), and (35), the following result holds:

$$
\begin{aligned}
L V_{2} \leq & -k_{1} z_{1}^{4}+\rho_{1}+z_{2}^{3}\left(f_{2}\left(\bar{x}_{2}, x_{3}\right)+w_{2}\right) \\
& -\left(k_{2}+\frac{3}{2}\right) z_{2}^{4}+\frac{b_{m}}{\sigma} \widetilde{\theta}\left(\frac{\sigma}{2 a_{1}^{2}} z_{1}^{6}-\dot{\hat{\theta}}\right) \\
& -\frac{\partial \alpha_{1}}{\partial \widehat{\theta}} z_{2}^{3} \sum_{j=3}^{n} \frac{\sigma}{2 a_{j}^{2}} z_{j}^{6}+\frac{3}{4} l_{2}^{2},
\end{aligned}
$$

where

$$
\begin{aligned}
w_{2}= & \frac{b_{M}^{4}}{4} z_{2}-\frac{\partial \alpha_{1}}{\partial x_{1}} f_{2}\left(x_{1}, x_{2}\right) \\
& -\sum_{j=0}^{1} \frac{\partial \alpha_{1}}{\partial y_{d}^{(j)}} y_{d}^{(j+1)}-\frac{1}{2} \frac{\partial^{2} \alpha_{1}}{\partial x_{1}^{2}} h_{1}^{T} h_{1} \\
& +\frac{3}{4} l_{2}^{-2} z_{2}\left\|h_{2}-\frac{\partial \alpha_{1}}{\partial x_{1}} h_{1}\right\|^{4} \\
& -\frac{\partial \alpha_{1}}{\partial \hat{\theta}}\left(\sum_{j=1}^{2} \frac{\sigma}{2 a_{j}^{2}} z_{j}^{6}-\sigma \hat{\theta}\right)+\left(k_{2}+\frac{3}{2}\right) z_{2} .
\end{aligned}
$$

It can be seen from (37) that $\partial w_{2} / \partial x_{3}=0$. Then, from Assumption 5, we have

$$
\frac{\partial\left[f_{2}\left(\bar{x}_{2}, x_{3}\right)+w_{2}\right]}{\partial x_{3}} \geq b_{m}>0 .
$$


According to Lemma 1 in [37], there exists a smooth ideal control input $x_{3}=\bar{\alpha}_{2}\left(\bar{x}_{2}, w_{2}\right)$ such that

$$
f_{2}\left(\bar{x}_{2}, \bar{\alpha}_{2}\right)+w_{2}=0
$$

By applying mean value theorem [48], there exists $\mu_{2}(0<$ $\left.\mu_{2}<1\right)$ such that

$$
f_{2}\left(\bar{x}_{2}, x_{3}\right)=f_{2}\left(\bar{x}_{2}, \bar{\alpha}_{2}\right)+g_{\mu_{2}}\left(x_{3}-\bar{\alpha}_{2}\right),
$$

where $g_{\mu_{2}}=\left.\left(\partial f_{2}\left(\bar{x}_{2}, x_{3}\right) / \partial x_{3}\right)\right|_{x_{3}=x_{\mu_{2}}}, x_{\mu_{2}}=\mu_{2} x_{3}+\left(1-\mu_{2}\right) \bar{\alpha}_{2}$. Assumption 5 on $g_{2}\left(\bar{x}_{2}, x_{3}\right)$ is still valid for $g_{\mu_{2}}$.

Substituting (39) and (40) into (36) produces

$$
\begin{aligned}
L V_{2} \leq & -k_{1} z_{1}^{4}+\rho_{1}+z_{2}^{3} g_{\mu_{2}} z_{3}+z_{2}^{3} g_{\mu_{2}}\left(\alpha_{2}-\bar{\alpha}_{2}\right) \\
& -\left(k_{2}+\frac{3}{2}\right) z_{2}^{4}+\frac{b_{m}}{\sigma} \widetilde{\theta}\left(\frac{\sigma}{2 a_{1}^{2}} z_{1}^{6}-\dot{\hat{\theta}}\right) \\
& -\frac{\partial \alpha_{1}}{\partial \widehat{\theta}} z_{2}^{3} \sum_{j=3}^{n} \frac{\sigma}{2 a_{j}^{2}} z_{j}^{6}+\frac{3}{4} l_{2}^{2},
\end{aligned}
$$

where $z_{3}=x_{3}-\alpha_{2}$.

Further, fuzzy logic system $W_{2}^{T} S\left(Z_{2}\right)$ is used to approximate the desired unknown virtual signal $\bar{\alpha}_{i}$ over a compact set $\Omega_{Z_{2}} \subset R^{6}$ such that, for any given positive constant $\varepsilon_{i}, \bar{\alpha}_{2}$ can be shown as

$$
\bar{\alpha}_{2}=W_{2}^{T} S_{2}\left(Z_{2}\right)+\delta_{2}\left(Z_{2}\right), \quad\left|\delta_{2}\left(Z_{2}\right)\right| \leq \varepsilon_{2},
$$

with $\delta_{2}\left(Z_{2}\right)$ being the approximation error.

Then, constructing the virtual control signal $\alpha_{2}$ in (13) and repeating the same methods used in (26) and (27), one has

$$
\begin{gathered}
-z_{2}^{3} g_{\mu_{2}} \bar{\alpha}_{2} \leq \frac{b_{m}}{2 a_{2}^{2}} z_{2}^{6} \theta+\frac{1}{2} a_{2}^{2}+\frac{3}{4} z_{2}^{4}+\frac{1}{4} b_{M}^{4} \varepsilon_{2}^{4}, \\
z_{2}^{3} g_{\mu_{2}} \alpha_{2} \leq-\frac{b_{m}}{2 a_{2}^{2}} z_{2}^{6} \widehat{\theta} .
\end{gathered}
$$

By taking (43) into account and using Young's inequality to the term $z_{2}^{3} g_{\mu_{2}} z_{3}$, (41) can be rewritten as

$$
\begin{aligned}
L V_{2} \leq & \sum_{j=1}^{2}\left(-k_{j} z_{j}^{4}+\rho_{j}\right)+\frac{b_{m} \tilde{\theta}}{\sigma}\left(\sum_{j=1}^{2} \frac{\sigma}{2 a_{j}^{2}} z_{j}^{6}-\dot{\hat{\theta}}\right) \\
& -\frac{\partial \alpha_{1}}{\partial \widehat{\theta}} z_{2}^{3} \sum_{j=3}^{n} \frac{\sigma}{2 a_{j}^{2}} z_{j}^{6}+\frac{1}{4} b_{M}^{4} z_{3}^{4},
\end{aligned}
$$

where $\rho_{j}=(1 / 2) a_{j}^{2}+(1 / 4) b_{M}^{4} \varepsilon_{j}^{4}+(3 / 4) l_{j}^{2}, \quad j=1,2$.

Remark 9. The adaptive law $\dot{\hat{\theta}}$ defined in (14) is a function of all the error variables. So, unlike the conventional approximation-based adaptive control schemes, $\left(\partial \alpha_{1} / \partial \hat{\theta}\right) \dot{\hat{\theta}}$ in (30) cannot be approximated directly by fuzzy logic system $W_{2}^{T} S_{2}\left(Z_{2}\right)$. To solve this problem, in (35), $\left(\partial \alpha_{1} / \partial \widehat{\theta}\right) \dot{\hat{\theta}}$ is divided into two parts. The first term in the right hand side of (35) is contained in $\bar{\alpha}_{2}$ to be modeled by $W_{2}^{T} S_{2}\left(Z_{2}\right)$, and the last term in (35) which is a function of the latter error variables, namely, $z_{j}, j=3,4, \ldots, n$, will be dealt with in the later design steps. This design idea will be repeated at the following steps.

Step $i(3 \leq i \leq n-1)$. From $z_{i}=x_{i}-\alpha_{i-1}$ and Itô formula, we have

$$
d z_{i}=\left(f_{i}\left(\bar{x}_{i}, x_{i+1}\right)-\ell \alpha_{i-1}\right) d t+\left(h_{i}-\sum_{j=1}^{i-1} \frac{\partial \alpha_{i-1}}{\partial x_{j}} h_{j}\right)^{T} d w
$$

where

$$
\begin{aligned}
\ell \alpha_{i-1}= & \sum_{j=1}^{i-1} \frac{\partial \alpha_{i-1}}{\partial x_{j}} f_{j}\left(\bar{x}_{j}, x_{j+1}\right)+\sum_{j=0}^{i-1} \frac{\partial \alpha_{i-1}}{\partial y_{d}^{(j)}} y_{d}^{(j+1)} \\
& +\frac{\partial \alpha_{i-1}}{\partial \widehat{\theta}} \dot{\hat{\theta}}+\frac{1}{2} \sum_{p, q=1}^{i-1} \frac{\partial^{2} \alpha_{i-1}}{\partial x_{p} \partial x_{q}} h_{q}^{T} h_{p} .
\end{aligned}
$$

Choose a Lyapunov function as

$$
V_{i}=V_{i-1}+\frac{1}{4} z_{i}^{4}
$$

Then, it follows that

$$
\begin{aligned}
L V_{i}= & L V_{i-1}+z_{i}^{3}\left(f_{i}\left(\bar{x}_{i}, x_{i+1}\right)-\ell \alpha_{i-1}\right) \\
& +\frac{3}{2} z_{i}^{2}\left(h_{i}-\sum_{j=1}^{i-1} \frac{\partial \alpha_{i-1}}{\partial x_{j}} h_{j}\right)^{T} \\
& \times\left(h_{i}-\sum_{j=1}^{i-1} \frac{\partial \alpha_{i-1}}{\partial x_{j}} h_{j}\right)
\end{aligned}
$$

where the term $L V_{i-1}$ in (48) can be obtained in the following form by straightforward derivations as those in former steps:

$$
\begin{aligned}
L V_{i-1} \leq & \sum_{j=1}^{i-1}\left(-k_{j} z_{j}^{4}+\rho_{j}\right)+\frac{b_{m} \tilde{\theta}}{\sigma}\left(\sum_{j=1}^{i-1} \frac{\sigma}{2 a_{j}^{2}} z_{j}^{6}-\dot{\hat{\theta}}\right) \\
& -\sum_{m=1}^{i-2} \frac{\partial \alpha_{m}}{\partial \widehat{\theta}} z_{m+1}^{3} \sum_{j=i}^{n} \frac{\sigma}{2 a_{j}^{2}} z_{j}^{6}+\frac{b_{M}^{4}}{4} z_{i}^{4},
\end{aligned}
$$

where $\rho_{j}=(1 / 2) a_{j}^{2}+(1 / 4) b_{M}^{4} \varepsilon_{j}^{4}+(3 / 4) l_{j}^{2}$.

According to the definition of $\dot{\hat{\theta}}$ in (14), one has

$$
\frac{\partial \alpha_{i-1}}{\partial \widehat{\hat{\theta}}}=\frac{\partial \alpha_{i-1}}{\partial \widehat{\theta}}\left(\sum_{j=1}^{i} \frac{\sigma}{2 a_{j}^{2}} z_{j}^{6}-\gamma \widehat{\theta}\right)+\frac{\partial \alpha_{i-1}}{\partial \widehat{\theta}} \sum_{j=i+1}^{n} \frac{\sigma}{2 a_{j}^{2}} z_{j}^{6}
$$


Then, substituting (49) into (48) and taking (50) into account, (48) can be rewritten as

$$
\begin{aligned}
L V_{i} \leq & \sum_{j=1}^{i-1}\left(-k_{j} z_{j}^{4}+\rho_{j}\right)+z_{i}^{3}\left(f_{i}\left(\bar{x}_{i}, x_{i+1}\right)+w_{i}\right) \\
& -\left(k_{i}+\frac{3}{2}\right) z_{i}^{4}+\frac{b_{m} \tilde{\theta}}{\sigma}\left(\sum_{j=1}^{i-1} \frac{\sigma}{2 a_{j}^{2}} z_{j}^{6}-\dot{\hat{\theta}}\right) \\
& -\sum_{m=1}^{i-1} \frac{\partial \alpha_{m}}{\partial \hat{\theta}} z_{m+1}^{3} \sum_{j=i+1}^{n} \frac{\sigma}{2 a_{j}^{2}} z_{j}^{6}+\frac{3}{4} l_{i}^{2},
\end{aligned}
$$

where

$$
\begin{aligned}
w_{i}= & \frac{b_{M}^{4}}{4} z_{i}-\sum_{j=1}^{i-1} \frac{\partial \alpha_{i-1}}{\partial x_{j}} f_{j}\left(\bar{x}_{j}, x_{j+1}\right) \\
& -\sum_{j=0}^{i-1} \frac{\partial \alpha_{i-1}}{\partial y_{d}^{(j)}} y_{d}^{(j+1)}-\frac{1}{2} \sum_{p, q=1}^{i-1} \frac{\partial^{2} \alpha_{i-1}}{\partial x_{p} \partial x_{q}} h_{p}^{T} h_{q} \\
& +\frac{3}{4} l_{i}^{-2} z_{i}\left\|h_{i}-\sum_{j=1}^{i-1} \frac{\partial \alpha_{i-1}}{\partial x_{j}} h_{j}\right\|^{4} \\
& -\frac{\partial \alpha_{i-1}}{\partial \hat{\theta}}\left(\sum_{j=1}^{i} \frac{\sigma}{2 a_{j}^{2}} z_{j}^{6}-\sigma \widehat{\theta}\right) \\
& -\frac{\sigma}{2 a_{i}^{2}} z_{i}^{3} \sum_{m=1}^{i-2} \frac{\partial \alpha_{m}}{\partial \widehat{\theta}} z_{m+1}^{3}+\left(k_{i}+\frac{3}{2}\right) z_{i} .
\end{aligned}
$$

Noting $\partial w_{i} / \partial x_{i+1}=0$, it follows that

$$
\frac{\partial\left[f_{i}\left(\bar{x}_{i}, x_{i+1}\right)+w_{i}\right]}{\partial x_{i+1}} \geq b_{m}>0 .
$$

Based on Lemma 1 in [37], there exists a smooth ideal control input $x_{i+1}=\bar{\alpha}_{i}\left(\bar{x}_{i}, w_{i}\right)$ which makes

$$
f_{i}\left(\bar{x}_{i}, \bar{\alpha}_{i}\right)+w_{i}=0
$$

In addition, applying mean value theorem [48], there exists $\mu_{i}\left(0<\mu_{i}<1\right)$ such that

$$
f_{i}\left(\bar{x}_{i}, x_{i+1}\right)=f_{i}\left(\bar{x}_{i}, \bar{\alpha}_{i}\right)+g_{\mu_{i}}\left(x_{i+1}-\bar{\alpha}_{i}\right)
$$

where $g_{\mu_{i}}=\left.\left(\partial f_{i}\left(\bar{x}_{i}, x_{i+1}\right) / \partial x_{i+1}\right)\right|_{x_{i+1}=x_{\mu_{i}}}, x_{\mu_{i}}=\mu_{i} x_{i+1}+(1-$ $\left.\mu_{i}\right) \bar{\alpha}_{i}$. Assumption 5 on $g_{i}\left(\bar{x}_{i}, x_{i+1}\right)$ is still valid for $g_{\mu_{i}}$. Combining (51)-(55) yields

$$
\begin{aligned}
L V_{i} \leq & \sum_{j=1}^{i-1}\left(-k_{j} z_{j}^{4}+\rho_{j}\right)+z_{i}^{3} g_{\mu_{i}} z_{i+1}+z_{i}^{3} g_{\mu_{i}}\left(\alpha_{i}-\bar{\alpha}_{i}\right) \\
& -\left(k_{i}+\frac{3}{2}\right) z_{i}^{4}+\frac{b_{m} \widetilde{\theta}}{\sigma}\left(\sum_{j=1}^{i-1} \frac{\sigma}{2 a_{j}^{2}} z_{j}^{6}-\dot{\hat{\theta}}\right) \\
& -\sum_{m=1}^{i-1} \frac{\partial \alpha_{m}}{\partial \widehat{\theta}} z_{m+1}^{3} \sum_{j=i+1}^{n} \frac{\sigma}{2 a_{j}^{2}} z_{j}^{6}+\frac{3}{4} l_{i}^{2},
\end{aligned}
$$

where $z_{i+1}=x_{i+1}-\alpha_{i}$. Subsequently, using fuzzy logic system $W_{i}^{T} S\left(Z_{i}\right)$ to model $\bar{\alpha}_{i}$ over a compact set $\Omega_{Z_{i}} \subset R^{2 i+2}$ such that for any given $\varepsilon_{i}>0, \bar{\alpha}_{i}$ can be expressed as

$$
\bar{\alpha}_{i}=W_{i}^{T} S_{i}\left(Z_{i}\right)+\delta_{i}\left(Z_{i}\right), \quad\left|\delta_{i}\left(Z_{i}\right)\right| \leq \varepsilon_{i},
$$

with $\delta_{i}\left(Z_{i}\right)$ being the approximation error. Furthermore, constructing virtual control signal $\alpha_{i}$ in (13) and following the same line as the procedures used from (26) to (27), one has

$$
\begin{gathered}
-z_{i}^{3} g_{\mu_{i}} \bar{\alpha}_{i} \leq \frac{b_{m}}{2 a_{i}^{2}} z_{i}^{6} \theta+\frac{1}{2} a_{i}^{2}+\frac{3}{4} z_{i}^{4}+\frac{1}{4} b_{M}^{4} \varepsilon_{i}^{4}, \\
z_{i}^{3} g_{\mu_{i}} \alpha_{i} \leq-\frac{b_{m}}{2 a_{i}^{2}} z_{i}^{6} \widehat{\theta} .
\end{gathered}
$$

By substituting (58) into (56) and using Young's inequality, we have

$$
\begin{aligned}
L V_{i} \leq & \sum_{j=1}^{i}\left(-k_{j} z_{j}^{4}+\rho_{j}\right)+\frac{b_{m} \tilde{\theta}}{\sigma}\left(\sum_{j=1}^{i} \frac{\sigma}{2 a_{j}^{2}} z_{j}^{6}-\dot{\hat{\theta}}\right) \\
& -\sum_{m=1}^{i-1} \frac{\partial \alpha_{m}}{\partial \widehat{\theta}} z_{m+1}^{3} \sum_{j=i+1}^{n} \frac{\sigma}{2 a_{j}^{2}} z_{j}^{6}+\frac{1}{4} b_{M}^{4} z_{i+1}^{4},
\end{aligned}
$$

where $\rho_{j}=(1 / 2) a_{j}^{2}+(1 / 4) b_{M}^{4} \varepsilon_{j}^{4}+(3 / 4) l_{j}^{2}$.

Step $n$. In this step, the actual control input signal $u$ will be obtained. By $z_{n}=x_{n}-\alpha_{n-1}$ and Itô formula, we have

$$
d z_{n}=\left(f_{n}\left(\bar{x}_{n}, u\right)-\ell \alpha_{n-1}\right) d t+\left(h_{n}-\sum_{j=1}^{n-1} \frac{\partial \alpha_{n-1}}{\partial x_{j}} h_{j}\right)^{T} d w
$$

where $\ell \alpha_{n-1}$ is defined in (46) with $i=n$. Choosing the Lyapunov function

$$
V_{n}=V_{n-1}+\frac{1}{4} z_{n}^{4}
$$

then, the following inequality can be easily verified by using (2), the completion of squares, and taking (59) with $i=n-1$ into account:

$$
\begin{aligned}
L V_{n} \leq & \sum_{j=1}^{n-1}\left(-k_{j} z_{j}^{4}+\rho_{j}\right)+\frac{b_{m} \tilde{\theta}}{\sigma}\left(\sum_{j=1}^{n-1} \frac{\sigma}{2 a_{j}^{2}} z_{j}^{6}-\dot{\hat{\theta}}\right) \\
& +z_{n}^{3}\left(f_{n}\left(\bar{x}_{n}, u\right)+w_{n}\right)-\left(k_{n}+\frac{3}{4}\right) z_{n}^{4}+\frac{3}{4} l_{n}^{2},
\end{aligned}
$$

where

$$
\begin{aligned}
w_{n}= & \ell \alpha_{n-1}-\sum_{m=1}^{n-2} \frac{\partial \alpha_{m}}{\partial \widehat{\theta}} z_{m+1}^{3} \frac{\sigma}{2 a_{n}^{2}} z_{n}^{3}+\frac{1}{4} b_{M}^{4} z_{n} \\
& +\frac{3}{4} l_{n}^{-2} z_{n}\left\|h_{n}-\sum_{j=1}^{n-1} \frac{\partial \alpha_{n-1}}{\partial x_{j}} h_{j}\right\|^{4}+\left(k_{n}+\frac{3}{4}\right) z_{n} .
\end{aligned}
$$


From the definition of $w_{n}$, Assumption 5 and Lemma 1 in [37], for every value $\bar{x}_{n}$ and $w_{n}$, there exists a smooth ideal control input $u=\bar{\alpha}_{n}\left(\bar{x}_{n}, w_{n}\right)$ such that $f_{n}\left(\bar{x}_{n}, \bar{\alpha}_{n}\right)+w_{n}=0$. By using mean value theorem [48], there exists $\mu_{n}\left(0<\mu_{n}<1\right)$ such that

$$
f_{n}\left(\bar{x}_{n}, u\right)=f_{i}\left(\bar{x}_{n}, \bar{\alpha}_{n}\right)+g_{\mu_{n}}\left(u-\bar{\alpha}_{n}\right)
$$

where $g_{\mu_{n}}=\left.\left(\partial f_{n}\left(\bar{x}_{n}, u\right) / \partial u\right)\right|_{u=x_{\mu_{n}}}, x_{\mu_{n}}=\mu_{n} u+\left(1-\mu_{n}\right) \bar{\alpha}_{n}$.

Furthermore, (62) can be rewritten as

$$
\begin{aligned}
L V_{n} \leq & \sum_{j=1}^{n-1}\left(-k_{j} z_{j}^{4}+\rho_{j}\right)+\frac{b_{m} \tilde{\theta}}{\sigma}\left(\sum_{j=1}^{n-1} \frac{\sigma}{2 a_{j}^{2}} z_{j}^{6}-\dot{\hat{\theta}}\right) \\
& +z_{n}^{3} g_{\mu_{n}}\left(u-\bar{\alpha}_{n}\right)-\left(k_{n}+\frac{3}{4}\right) z_{n}^{4}+\frac{3}{4} l_{n}^{2} .
\end{aligned}
$$

Next, using fuzzy logic system $W_{n}^{T} S\left(Z_{n}\right)$ to approximate the unknown function $\bar{\alpha}_{n}$ on the compact set $\Omega_{Z_{n}} \subset R^{2 n+2}$, constructing the actual control $u$ in (13) with $i=n$, and repeating the same procedures as (58)-(59), one has

$$
L V_{n} \leq \sum_{j=1}^{n}\left(-k_{j} z_{j}^{4}+\rho_{j}\right)+\frac{b_{m} \tilde{\theta}}{\sigma}\left(\sum_{j=1}^{n} \frac{\sigma}{2 a_{j}^{2}} z_{j}^{6}-\dot{\hat{\theta}}\right) .
$$

Further, choosing the adaptive law $\dot{\hat{\theta}}$ in (14) and using the inequality $\left(\gamma b_{m} / \sigma\right) \tilde{\theta} \hat{\theta} \leq-\left(\gamma b_{m} / 2 \sigma\right) \widetilde{\theta}^{2}+\left(\gamma b_{m} / 2 \sigma\right) \theta^{2}$ result in

$$
L V_{n} \leq-\sum_{j=1}^{n} k_{j} z_{j}^{4}-\frac{\gamma b_{m}}{2 \sigma} \widetilde{\theta}^{2}+\sum_{j=1}^{n} \rho_{j}+\frac{\gamma b_{m}}{2 \sigma} \theta^{2}
$$

where $\rho_{j}=(1 / 2) a_{j}^{2}+(1 / 4) b_{M}^{4} \varepsilon_{j}^{4}+(3 / 4) l_{j}^{2}, j=1,2, \ldots, n$.

Now, the actual control signal $u$ is constructed. The main result will be summarized by the following theorem.

Theorem 10. Consider the pure-feedback stochastic nonlinear system (5), the controller (13), and adaptive law (14) under Assumptions 5 and 6 . Assume that there exists sufficiently large compacts $\Omega_{Z_{i}}, i=1,2, \ldots, n$, such that $Z_{i} \in \Omega_{Z_{i}}$, for all $t \geq 0$; then, for bounded initial conditions $\left[\bar{z}_{n}(0)^{T}, \hat{\theta}(0)\right]^{T} \in \Omega_{0}$ (where $\Omega_{0}$ is an appropriately chosen compact set),

(i) all the signals in the closed-loop system are bounded in probability;

(ii) there exists a finite time $T_{1}$ such that the quartic mean square tracking error enters inside the area for all $t>$ $T_{1}$,

$$
\Omega_{1}=\left\{y(t) \in R \mid E\left[\left|y-y_{d}\right|^{4}\right] \leq 8 \rho, \forall t>T_{1}\right\},
$$

where the time $T_{1}$ will be given later.

Proof. (i) For the stability analysis of the closed-loop system, choose the Lyapunov function as $V=V_{n}$. From (67), it follows that

$$
L V \leq-a_{0} V+b_{0}
$$

where $a_{0}=\min \left\{4 k_{j}, \gamma, j=1,2, \ldots, n\right\}$ and $b_{0}=\sum_{j=1}^{n} \rho_{j}+$ $\left(\gamma b_{m} / 2 \sigma\right) \theta^{2}$. Therefore, based on Lemma 4 in [37], $z_{j}$ and $\tilde{\theta}$ remain bounded in probability. Because $\theta$ is a constant, $\widehat{\theta}$ is bounded in probability. It can be further seen that $\alpha_{j}$ is a function of $z_{j}$ and $\hat{\theta}$. So, $\alpha_{j}$ is also bounded in probability. Hence, we conclude that all the signals $x_{j}$ in the closed-loop system (5) remain bounded in the sense of probability.

(ii) From (69), the following inequality can be obtained directly by [24, Theorem 4.1]

$$
\frac{d E[V(t)]}{d t} \leq-a_{0} E[V(t)]+b_{0} .
$$

Let $\rho:=b_{0} / a_{0}>0$, then (70) satisfies

$$
0 \leq E[V(t)] \leq V(0) e^{-a_{0} t}+\rho .
$$

Then, it can be easily verified that there exists a time $T_{1}=$ $\max \left\{0,\left(1 / a_{0}\right) \ln (V(0) / \rho)\right\}$ such that

$$
E\left[\left|y-y_{d}\right|^{4}\right] \leq 4 E[V(t)] \leq 8 \rho, \quad \forall t>T_{1} .
$$

\section{Simulation Results}

In this section, to illustrate the effectiveness of the proposed control scheme, we consider the following second-order pure-feedback stochastic nonlinear system:

$$
\begin{gathered}
d x_{1}=\left(x_{2}+0.05 \sin \left(x_{2}\right)\right) d t+0.1 \cos \left(x_{1}\right) d w \\
d x_{2}=\left(\left(2+\frac{x_{1}^{2}}{1+x_{1}^{2}+x_{2}^{2}}\right) u+0.1 u^{3}\right) d t \\
+0.2 \sin \left(x_{1} x_{2}\right) d w \\
y=x_{1} .
\end{gathered}
$$

The control objective is to design an adaptive controller for the system such that all the signals in the closed-loop system remain bounded and the system output $y$ tracks the given reference signal $y_{d}=0.5(\sin (t)+\sin (0.5 t))$. According to Theorem 10, choose the virtual control law $\alpha_{1}$ in (13), actual control input $u$ in (13) with $i=2$, and adaptive law in (14). The design parameters are taken as follows: $a_{1}=a_{2}=0.06$, $\gamma=0.0015$, and $\sigma=25$. The initial conditions are given by $\left[x_{1}(0), x_{2}(0)\right]^{T}=[0.2,0.5]^{T}$ and $\hat{\theta}(0)=0$.

The simulation results are shown in Figures 1-4. Figure 1 shows the system output $y$ and the reference signal $y_{d}$. Figure 2 and Figure 3 show that the state variable $x_{2}$ and adaptive parameter $\hat{\theta}$ are bounded. Figure 4 displays the control input signal $u$.

\section{Conclusions}

In this paper, a novel fuzzy-based adaptive control scheme has been presented for pure-feedback stochastic nonlinear systems. The proposed controller guarantees that all the signals in the closed-loop systems are bounded in probability 


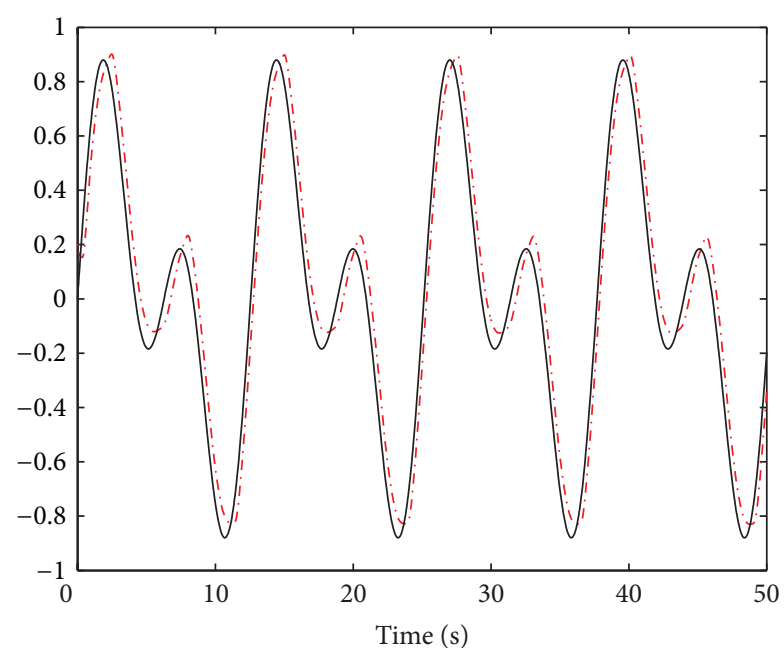

$-\cdot-x_{1}$

$-y_{d}$

FIGURE 1: System output $y$ and reference signal $y_{d}$.

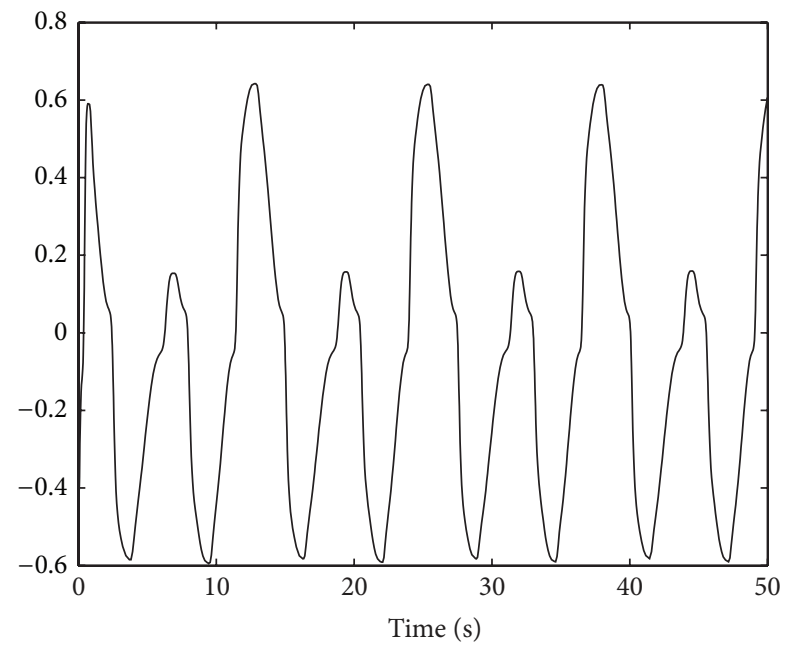

FIgURE 2: The state variable $x_{2}$.

and tracking error eventually converges to a small neighborhood around the origin in the sense of mean quartic value. The main advantages of this control scheme are that the controller is simpler than the existing ones and only one adaptive parameter needs to be estimated online for an $n$-order system. Numerical results have been provided to show the effectiveness of the suggested approach. Our future research will mainly focus on the multi-input and multioutput (MIMO) pure-feedback stochastic nonlinear systems based on the result in this paper.

\section{Conflict of Interests}

The authors declare that there is no conflict of interests regarding the publication of this paper.

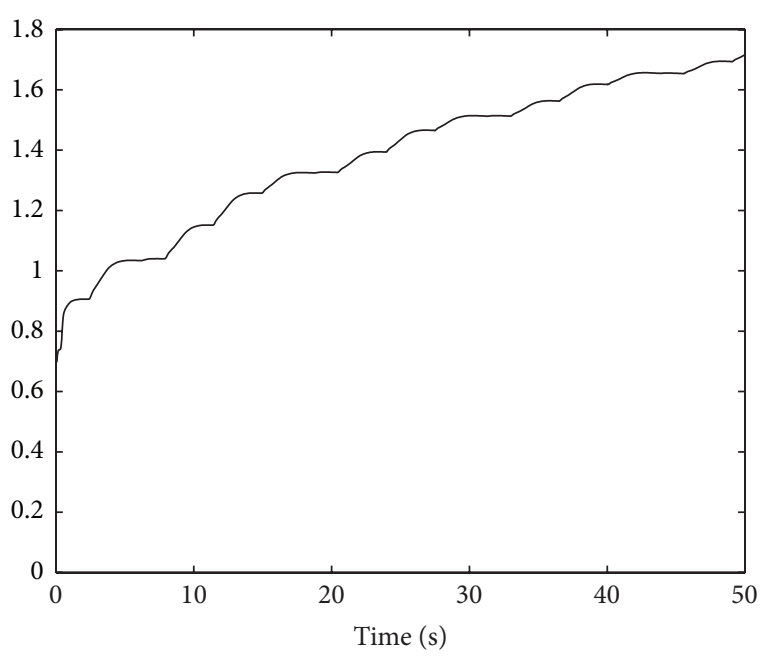

$-\hat{\theta}$

FIGURE 3: The adaptive parameter $\widehat{\theta}$.

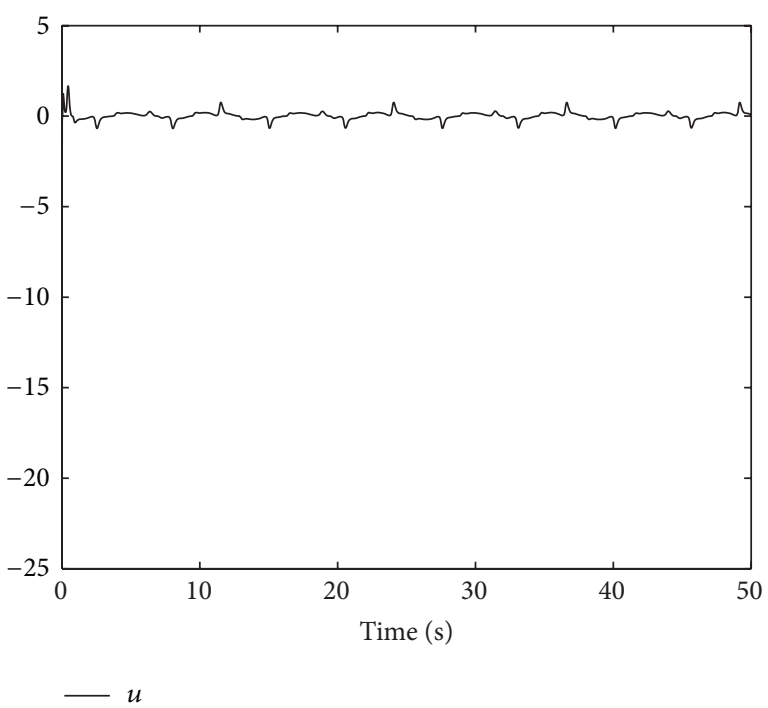

FIgURE 4: The true control input $u$.

\section{Acknowledgments}

This work is partially supported by the Natural Science Foundation of China (61304002, 61304003, and 11371071), the Program for New Century Excellent Tallents in University (NECT-13-0696), the Program for Liaoning Innovative Research Team in University under Grant (LT2013023), the Program for Liaoning Excellent Talents in University under Grant (LR2013053), and the Education Department of Liaoning Province under the general project research under Grant (no. L2013424).

\section{References}

[1] K. S. Narendra and A. M. Annaswamy, Stable Adaptive Systems, Prentice-Hall, Englewood Cliffs, NJ, USA, 1989. 
[2] H. Y. Li, J. Y. Yu, C. Hilton, and H. H. Liu, "Adaptive sliding mode control for nonlinear active suspension vehicle systems using T-S fuzzy Approach," IEEE Transactions on Industrial Electronics, vol. 60, no. 8, pp. 3328-3338, 2013.

[3] H. Y. Li, J. Y. Yu, C. Hilton, and H. H. Liu, "Adaptive sliding mode control for nonlinear active suspension vehicle systems using T-S fuzzy approach," IEEE Transactions on Industrial Electronics, vol. 60, no. 8, pp. 3328-3338, 2013.

[4] M. Krstic, I. Kanellakopoulos, and P. V. Kokotovic, Nonlinear and Adaptive Control Design, Wiley, New York, NY, USA, 1995.

[5] M. Liu, X. Cao, and P. Shi, "Fault estimation and tolerant control for fuzzy stochastic systems," IEEE Transactions on Fuzzy Systems, vol. 21, no. 2, pp. 221-2229, 2013.

[6] M. Liu, X. Cao, and P. Shi, "Fuzzy-model-based fault tolerant design for nonlinear stochastic systems against simultaneous sensor and actuator faults," IEEE Transactions on Fuzzy Systems, vol. 21, no. 5, pp. 789-799, 2013.

[7] S. Yin, S. Ding, and H. Luo, "Real-time implementation of fault tolerant control system with performance optimization," IEEE Transactions on Industrial Electronics, vol. 61, no. 5, pp. 24022411, 2013.

[8] S. Yin, S. Ding, A. Haghani, H. Hao, and P. Zhang, "A comparison study of basic data-driven fault diagnosis and process monitoring methods on the benchmark Tennessee Eastman process," Journal of Process Control, vol. 22, no. 9, pp. 1567-1581, 2012.

[9] H. Li, H. Liu, H. Gao, and P. Shi, "Reliable fuzzy control for active suspension systems with actuator delay and fault," IEEE Transactions on Fuzzy Systems, vol. 20, no. 2, pp. 342-357, 2012.

[10] H. Y. Li, X. J. Jing, H. K. Lam, and P. Shi, "Fuzzy Sampled-data control for uncertain vehicle suspension systems," IEEE Transactions on Cybernetics, 2013.

[11] H. Y. Li, X. J. Jing, and H. R. Karimi, "Output-feedback based h-infinity control for active suspension systems with control delay," IEEE Transactions on Industrial Electronics, vol. 61, no. 1, pp. 436-446, 2014.

[12] M. Chen, S. S. Ge, B. V. E. How, and Y. S. Choo, "Robust adaptive position mooring control for marine vessels," IEEE Transactions on Control Systems Technology, vol. 21, no. 2, pp. 395-409, 2013.

[13] S. Bououden, M. Chadli, F. Allouani, and S. Filali, "A new approach for fuzzy predictive adaptive controller design using particle swarm optimization algorithm," International Journal of Innovative Computing, Information and Control, vol. 9, no. 9, pp. 3741-3758, 2013.

[14] S. Sefriti, J. Boumhidi, M. Benyakhlef, and I. Boumhidi, "Adaptive decentralized sliding mode neural network control of a class of nonlinear interconnected systems," International Journal of Innovative Computing, Information and Control, vol. 9, no. 7, pp. 2941-2947, 2013.

[15] C. M. Lin, C. F. Hsu, and R. G. Yeh, "Adaptive fuzzy slidingmode control system design for brushless DC motors," International Journal of Innovative Computing, Information and Control, vol. 9, no. 3, pp. 1259-1270, 2013.

[16] T. Shaocheng, C. Bin, and W. Yongfu, "Fuzzy adaptive output feedback control for MIMO nonlinear systems," Fuzzy Sets and Systems, vol. 156, no. 2, pp. 285-299, 2005.

[17] B. Chen, X. Liu, K. Liu, and C. Lin, "Direct adaptive fuzzy control of nonlinear strict-feedback systems," Automatica, vol. 45, no. 6, pp. 1530-1535, 2009.

[18] B. Chen, X.-P. Liu, S.-C. Tong, and C. Lin, "Observer-based stabilization of T-S fuzzy systems with input delay," IEEE Transactions on Fuzzy Systems, vol. 16, no. 3, pp. 652-663, 2008.
[19] X. D. Zhao, L. X. Zhang, P. Shi, and H. R. Karimi, "Novel stability criteria for T-S fuzzy systems," IEEE Transactions on Fuzzy Systems, vol. 21, no. 6, pp. 1-11, 2013.

[20] X. J. Su, P. Shi, L. Wu, and Y. Song, "A novel approach to filter design for T-S fuzzy discretetime systems with time-varying delay," IEEE Transactions on Fuzzy Systems, vol. 20, no. 6, pp. 1114-1129, 2012.

[21] X. J. Su, P. Shi, L. Wu, and Y. -D. Song, "A novel control design on discrete-time Takagi- Sugeno fuzzy systems with time-varying delays," IEEE Trans on Fuzzy Systems, vol. 20, no. 6, pp. 655-671, 2013.

[22] X. J. Su, P. Shi, L. Wu, and S. K. Nguang, "Induced $\ell 2$ filtering of fuzzy stochastic systems with time-varying delays," IEEE Transactions on Cybernetics, vol. 43, no. 4, pp. 1251-1264, 2013.

[23] H. Deng and M. Krstić, "Stochastic nonlinear stabilization. I. A backstepping design," Systems \& Control Letters, vol. 32, no. 3, pp. 143-150, 1997.

[24] H. Deng, M. Krstić, and R. J. Williams, "Stabilization of stochastic nonlinear systems driven by noise of unknown covariance," IEEE Transactions on Automatic Control, vol. 46, no. 8, pp. 12371253, 2001.

[25] S.-J. Liu, J.-F. Zhang, and Z.-P. Jiang, "Decentralized adaptive output-feedback stabilization for large-scale stochastic nonlinear systems," Automatica, vol. 43, no. 2, pp. 238-251, 2007.

[26] L. Liu and X.-J. Xie, "Output-feedback stabilization for stochastic high-order nonlinear systems with time-varying delay," Automatica, vol. 47, no. 12, pp. 2772-2779, 2011.

[27] L. Liu, N. Duan, and X.-J. Xie, “Output-feedback stabilization for stochastic high-order nonlinear systems with a ratio of odd integers power," Acta Automatica Sinica, vol. 36, no. 6, pp. 858864, 2010.

[28] Z. Wu, M. Cui, P. Shi, and H. R. Karimi, "Stability of stochastic nonlinear systems with state-dependent switching," IEEE Transactions on Automatic Control, vol. 58, no. 8, pp. 1904-1918, 2013.

[29] H. R. Karimi, "Robust delay-dependent $H_{\infty}$ control of uncertain time-delay systems with mixed neutral, discrete, and distributed time-delays and Markovian switching parameters," IEEE Transactions on Circuits and Systems. I, vol. 58, no. 8, pp. 1910-1923, 2011.

[30] S. Huang, Z. Xiang, and H. R. Karimi, "Robust $l_{2}$-gain control for $2 \mathrm{D}$ nonlinear stochastic systems with time-varying delays and actuator saturation," Journal of the Franklin Institute, vol. 350, no. 7, pp. 1865-1885, 2013.

[31] H. E. Psillakis and A. T. Alexandridis, "Adaptive neural tracking for a class of SISO uncertain and stochastic nonlinear systems," in Proceedings of the 44th IEEE Conference on Decision and Control, and the European Control Conference (CDC-ECC '05), pp. 7822-7827, Seville, Spain, December 2005.

[32] Q. Zhou, P. Shi, H. H. Liu, and S. Y. Xu, "Neural-network-based decentralized adaptive output-feedback control for large-scale stochastic nonlinear systems," IEEE Transactions on Systems, Man, and Cybernetics B, vol. 42, no. 6, pp. 1608-1619, 2012.

[33] Y. Li, S. C. Tong, and Y. M. Li, "Observer-based adaptive fuzzy backstepping control for strict-feedback stochastic nonlinear systems with time delays," International Journal of Innovative Computing, Information and Control, vol. 8, pp. 8103-8114, 2012.

[34] S. C. Tong, T. Wang, Y. M. Li, and B. Chen, "A combined backstepping and stochastic small- gain approach to robust adaptive fuzzy output feedback control," IEEE Transactions on Fuzzy Systems, vol. 21, no. 2, pp. 314-327, 2013. 
[35] H. Q. Wang, B. Chen, and C. Lin, "Adaptive neural tracking control for a class of stochastic nonlinear systems with unknown dead-zone," International Journal of Innovative Computing, Information and Control, vol. 9, no. 8, pp. 3257-3270, 2013.

[36] A. Ferrara and L. Giacomini, "Control of a class of mechanical systems with uncertainties via a constructive adaptive/second order VSC approach," Journal of Dynamic Systems, Measurement and Control, vol. 122, no. 1, pp. 33-39, 2000.

[37] S. S. Ge and C. Wang, "Adaptive NN control of uncertain nonlinear pure-feedback systems," Automatica, vol. 38, no. 4, pp. 671-682, 2002.

[38] D. Wang and J. Huang, "Adaptive neural network control for a class of uncertain nonlinear systems in pure-feedback form," Automatica, vol. 38, no. 8, pp. 1365-1372, 2002.

[39] C. Wang, D. J. Hill, S. S. Ge, and G. Chen, "An ISS-modular approach for adaptive neural control of pure-feedback systems," Automatica, vol. 42, no. 5, pp. 723-731, 2006.

[40] T. P. Zhang and S. S. Ge, "Adaptive dynamic surface control of nonlinear systems with unknown dead zone in pure feedback form," Automatica, vol. 44, no. 7, pp. 1895-1903, 2008.

[41] M. Wang, S. S. Ge, and K.-S. Hong, "Approximation-based adaptive tracking control of pure-feedback nonlinear systems with multiple unknown time-varying delays," IEEE Transactions on Neural Networks, vol. 21, no. 11, pp. 1804-1816, 2010.

[42] H. Q. Wang, B. Chen, X. P. Liu, K. F. Liu, and C. Lin, "Robust adaptive fuzzy tracking control for pure-feedback stochastic nonlinear systems with input constraints," IEEE Transaction on Cybernetics, vol. 43, no. 6, pp. 2093-2104, 2013.

[43] J. J. Yu, K. J. Zhang, and S. M. Fei, “Direct fuzzy tracking control of a class of nonaffine stochastic nonlinear systems with unknown dead-zone input," in Proceedings of the 17th IFAC World Congress, pp. 12236-12241, International Federation of Automatic Control (IFAC), Seoul, Korea, 2008.

[44] Z. Yu, Z. Jin, and H. Du, "Adaptive neural control for a class of non-affine stochastic non-linear systems with time-varying delay: a Razumikhin-Nussbaum method," IET Control Theory \& Applications, vol. 6, no. 1, pp. 14-23, 2012.

[45] Y. M. Li and S. C. Tong, "Adaptive fuzzy output-feedback control of pure-feedback uncertain nonlinear systems with unknown dead-zone," IEEE Transactions on Fuzzy Systems, 2013.

[46] S. C. Tong, Y. Li, Y. M. Li, and Y. J. Liu, “Observer-based adaptive fuzzy backstepping control for a class of stochastic nonlinear strict-feedback systems," IEEE Transactions on Systems, Man, and Cybernetics B, vol. 41, no. 6, pp. 1693-1704, 2011.

[47] L. X. Wang and J. M. Mendel, "Fuzzy basis functions, universal approximation, and orthogonal least squares learning," IEEE Transactions on Neural Networks, vol. 3, no. 5, pp. 807-814, 1992.

[48] T. M. Apostol, Mathematical Analysis, Addison-Wesley, Reading, Mass, USA, 1963. 


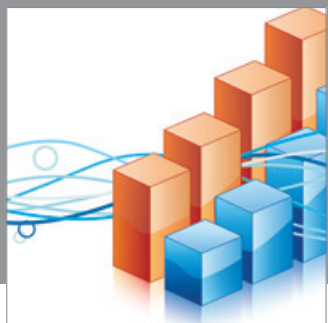

Advances in

Operations Research

mansans

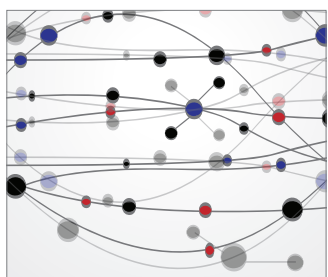

The Scientific World Journal
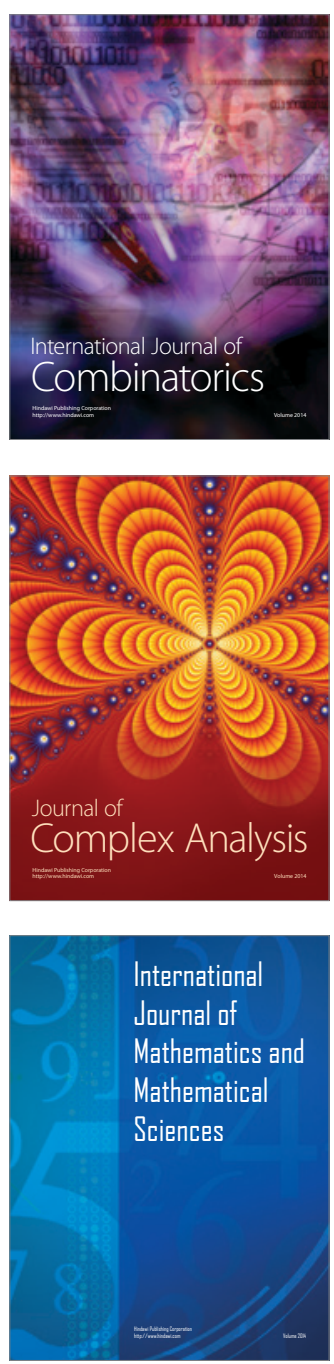
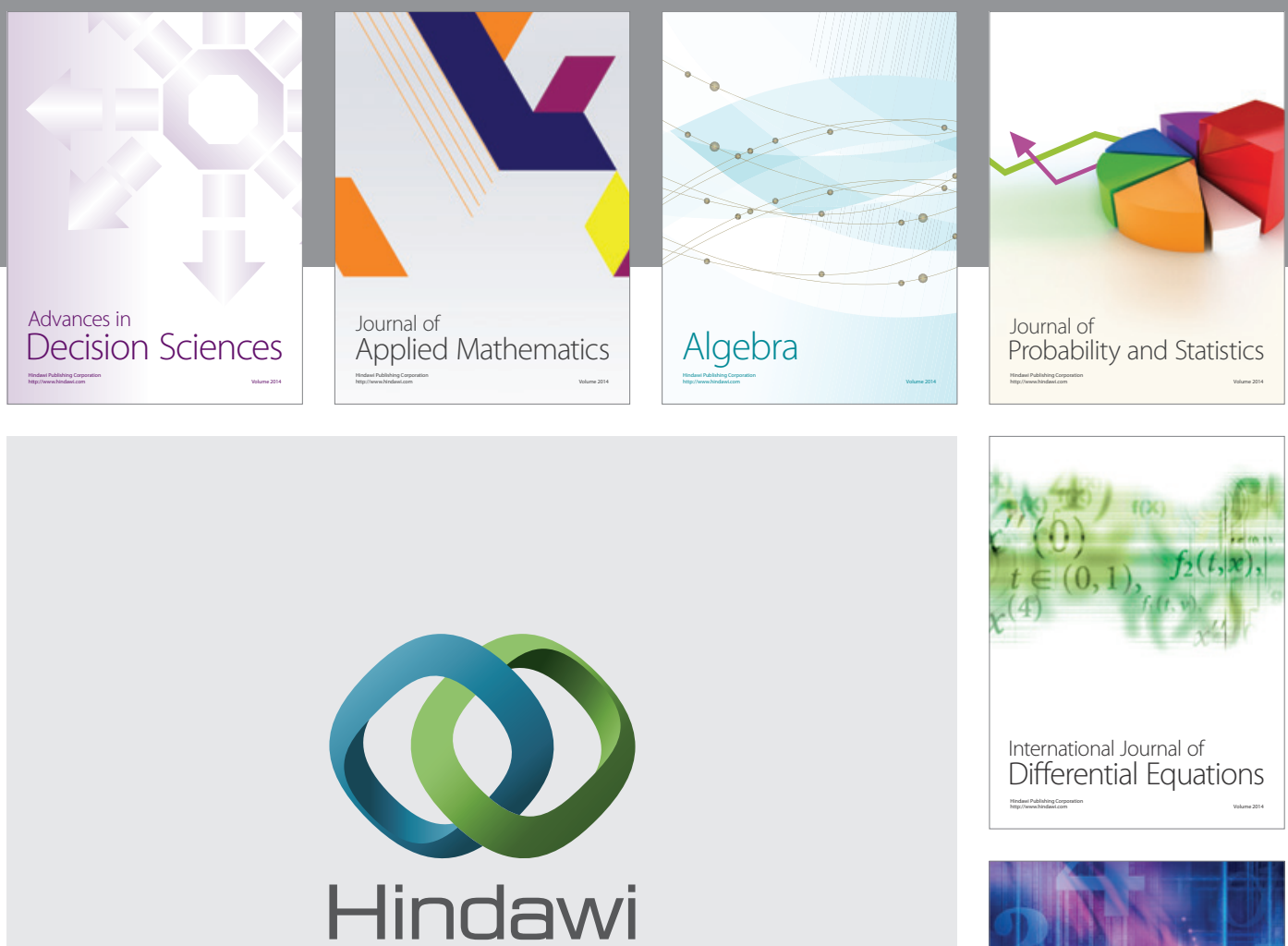

Submit your manuscripts at http://www.hindawi.com
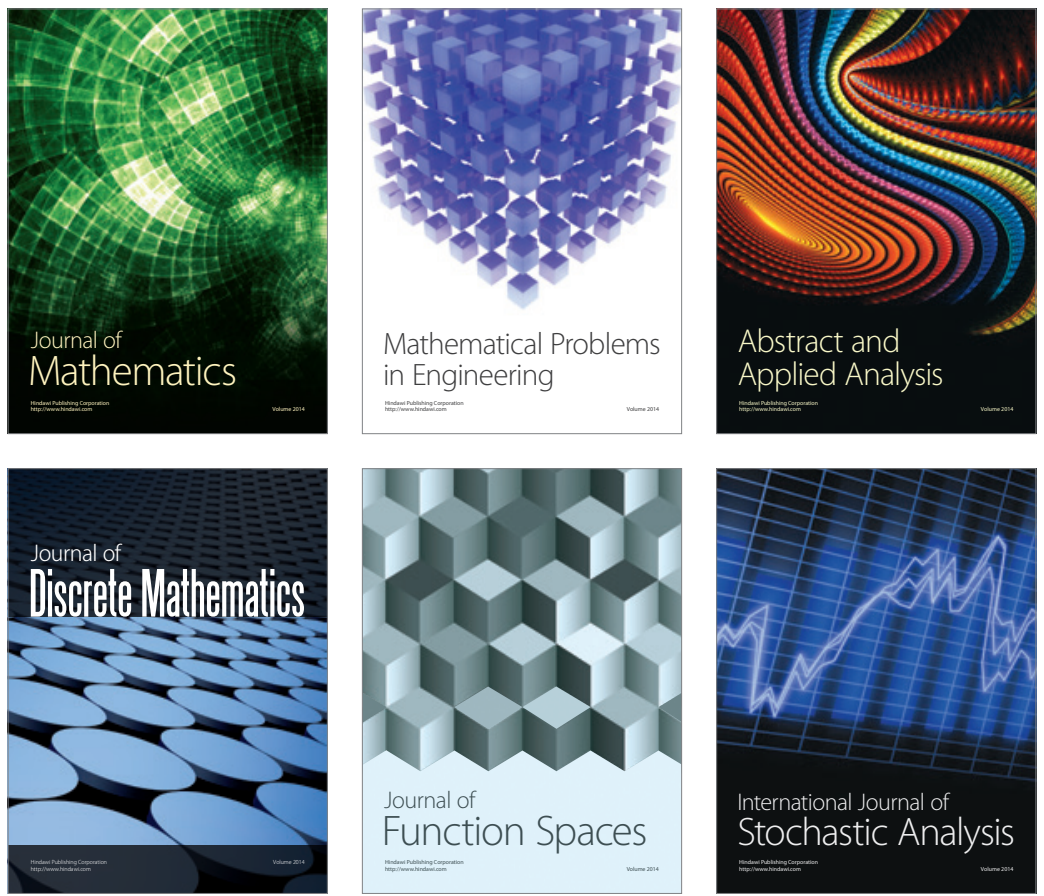

Journal of

Function Spaces

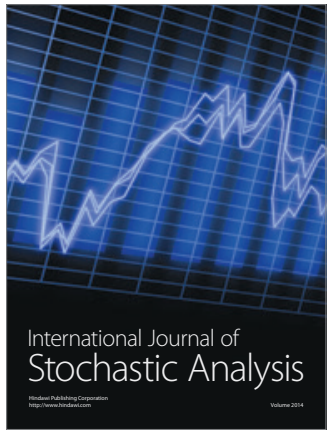

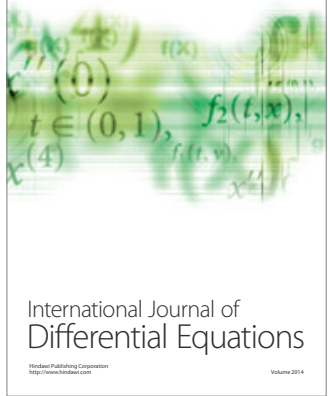
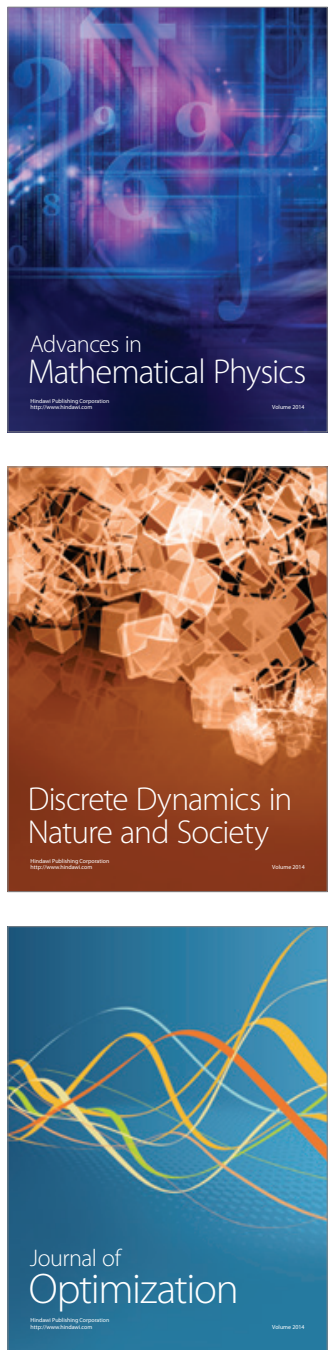